\title{
Zum Tod von Winfried Hassemer
}

Winfried Hassemer ist am 9. Januar 2014 gestorben. Strafrechtslehrer, Rechtsphilosoph, ehemaliger hessischer Datenschutzbeauftragter, Richter und Vizepräsident des Bundesverfassungsgericht, Anwalt und Rechtsgutachter, Ombudsmann für Verbraucher bei der Schufa Holding AG, Neutraler Mittler und Hinweisgeber bei Verdacht auf Regelwidrigkeiten im Unternehmen der Daimler AG: Alle diese vielseitigen Funktionen erfüllte Winfried Hassemer als liberaler Geist, stets mit dem Willen, auch schwierige Entscheidungen nachvollziehbar zu vermitteln.

Winfried Hassemer hat nicht nur den Datenschutz im Amt des hessischen Datenschutzbeauftragten geprägt, sondern auch die Querschnittsmaterie Datenschutz in ihren vielen Verästelungen mit seinen anderen Aufgaben verbunden. Davon zeugt bis heute eine Broschüre, die er 1995 zur Feier des 25 jährigen Jubiläums des Hessischen Datenschutzgesetzes herausgegeben hat. Dieses Büchlein enthält zwei seiner Vorträge zum Thema Datenschutz und Datenverarbeitung. Der erste Vortrag legt grundsätzliche schwierige Datenschutzfragen vereinfacht und beredt dar, der zweite befasst sich mit Konzept, Praxis und Politik des Datenschutzes bis hin zu bereichsspezifischen Beispielen etwa der Ermittlung im Strafprozess oder den Feldern der Polizei und Geheimdienste. So hat der Datenschützer und Strafrechtswissenschaftler die Lockerung der Grenzen zwischen Geheimdienst und Polizei immer wieder scharf kritisiert. Als Vizepräsident des Bundesverfassungsgerichts wirkte er an zahlreichen Entscheidungen mit, die sich mit den Sicherheitsmaßnahmen nach dem 11. September auseinandersetzten. Im Übrigen ist aus datenschutzrechtlicher Sicht die Entscheidung zum NPD-Verbotsverfahren hervorzuheben, in dem Bundesverfassungsgericht grundsätzliche Feststellungen zum Einsatz von nachrichtendienstlichen V-Leuten im Rahmen der Extremismusbekämpfung getroffen hat. Dabei hat sich Winfried Hassemer stets engagiert für eine substantielle Aufarbeitung der Extremismusproblematik ausgesprochen, wie seine Beiträge zu den NSU-Morden jüngst dokumentieren.

Der international hochgeachtete Gelehrte war Schüler des bekannten Münchner Rechtsphilosophen Arthur Kaufmanns, bei dem er den Willen zur Wahrheit bewunderte und seinen Kampf gegen Unrecht und Halbherzigkeit. Hassemer war wissenschaftlich global vernetzt und erhielt die Doktorwürde spanischer, portugiesischer, brasilianischer, sizilianischer und chinesischer Universitäten. Er war ein überzeugter Europäer, der nach dem Öffnen des Eisernen Vorhangs brillant und präzise die Sache Europas und des Datenschutzes etwa in Warschau und Budapest vertrat. Sein vielleicht letzter Beitrag lautete „Ach Europa“. Er schilderte darin seinen Traum von Europa und seine Trauer darüber, dass es der Ausstieg aus Europa bis auf die Agenda des öffentlichen Diskurses geschafft hat. Aber er versuchte auch einen Ausweg aus der Krise zu zeigen und betonte noch Ende 2013, dass das sich das Ziel einer Einheit Europas umso leichter erreichen und erhalten lässt, ,je fundamentaler diese Einheit - von der Kultur als Basis bis zur Wahrung als deren möglicher Konsequenz - loziert ist". Die gemeinsame europäische Rechtskultur sollte unter diesem Vorzeichen auch Basis einer gemeinsamen Datenschutzpolitik und Grundordnung sein.

Nicht nur im Datenschutz wird der Weggang von Winfried Hassemer eine empfindliche Lücke reißen.

\section{Thomas Petri, Marie-Theres Tinnefeld}

